\title{
Local electron and ionic heating effects on the conductance of nanostructures
}

\author{
Roberto D'Agosta and Massimiliano Di Ventra \\ Department of Physics, University of California - San Diego, La Jolla, CA 92093-0319
}

(Dated: October 31, 2018)

\begin{abstract}
Heat production and dissipation induced by current flow in nanostructures is of primary importance to understand the stability of these systems. These effects have contributions from both electron-phonon and electron-electron interactions. Here, we consider the effect of the local electron and ionic heating on the conductance of nanoscale systems. Specifically we show that the non-linear dependence of the conductance on the external bias may be used to infer information about the local heating of both electrons and ions. We compare our results with available experimental data on transport in $\mathrm{D}_{2}$ and $\mathrm{H}_{2}$ molecules. The comparison between experiment and theory is reasonably good close to the lowest phonon mode of the molecule, especially for the $\mathrm{D}_{2}$ molecule. At higher biases we cannot rule out the presence of other effects like, e.g., current-induced forces that make the scenario more complex.
\end{abstract}

\section{INTRODUCTION}

The idea of building electronic devices from nanostructures has gathered a lot of attention due to the high expectations in terms of size reduction and power dissipation [1]. Encouraging progress has been made in experimental techniques and theoretical modeling towards this aim [2]. However, a fundamental and technologically important issue, namely local heat production and dissipation in these systems has attracted much less attention [3, 4, 5, [6, 7, 8, 9 9, 10].

It has been argued that since the electron inelastic mean free path is large compared to the dimensions of a nanostructure, no energy dissipation occurs in the nanostructure region. However, nanoscale systems carry very large current densities compared to bulk electrodes. This implies an increased number of scattering events per unit time and unit volume so that interactions among electrons or among electrons and phonons are particularly important. In addition, the reduced size means a small heat capacitance: any small energy transfer from the current-carrying electrons to local ionic vibrations or other electrons in the system may induce a substantial heating of the nanostructure [7].

So far, direct measurements of the amount of energy locally dissipated in a nanoscale system have been beyond our reach. However, new experiments have considered the indirect effects of local heating on accessible quantities $[8,9,11,12]$. For example, in [8, 9] an effective ionic temperature is determined via the force needed to break the chemical bonds between molecules and the adjacent leads. These experiments indirectly probe the local ionic temperature, the contributions due to electron-electron interactions, and corresponding local electron heating [7, 9].

Here, we discuss another possible indirect method to probe both the local ionic and electron temperatures via the non-linearities in the DC conductance of nanostructures. We will compare our results with the experimental conductance of simple molecules such as $\mathrm{D}_{2}$ and $\mathrm{H}_{2}$ sandwiched between two Pt leads as studied in Ref. [13] (and references therein).

In order to address the above issues we need a theory that takes into account both energy production and dissipation on an equal footing. A full quantum-mechanical description in terms of many-body states for the present nonequilibrium problem seems hopeless. Instead, we have previously shown that a much more "economical" hydrodynamic theory in terms of the single-particle density and current density may be derived for nanostructures [14]. In this paper, we first review such a theory, and later on use it to study the effect of heating on conductance.

\section{CLASSICAL HYDRODYNAMICS}

In the following, we will refer to some concepts of classical hydrodynamics. For completeness, we repeat here some of those concepts, while a more comprehensive description of the dynamics of classical fluids can be found in many textbooks [15, 16].

The dynamics of a classical viscous fluid is usually described by the so-called Navier-Stokes equations for the single-particle density, $n(r, t)$, and the velocity field, $v(r, t)$, (ratio between the current density and the density)

$$
\begin{aligned}
D_{t} n(r, t) & =-n(r, t) \nabla \cdot v(r, t) \\
m n(r, t) D_{t} v_{i}(r, t)=-\nabla_{i} P(r, t)+\nabla_{j} \pi_{i, j}(r, t) & -n(r, t) \nabla_{i} V_{e x t}(r, t)
\end{aligned}
$$


where $P(r, t)$ is the pressure, $\pi_{i, j}(r, t)$ is the Navier-Stokes stress tensor

$$
\begin{aligned}
\pi_{i, j}(r, t)= & \eta\left[\nabla_{j} v_{i}(r, t)+\nabla_{i} v_{j}(r, t)-\frac{2}{3} \delta_{i, j} \nabla \cdot v(r, t)\right] \\
& +\zeta \delta_{i, j} \nabla \cdot v(r, t),
\end{aligned}
$$

and $V_{\text {ext }}(r, t)$ is the external potential. [Throughout the paper, $\nabla_{i}$ corresponds to the derivative with respect to the $i$-th spatial component $(i=\{x, y, z\})$, and summation over repeated indexes is understood.] In these equations, the operator $D_{t}=\partial_{t}+v(r, t) \cdot \nabla$ is the so-called "convective" derivative, while the viscosity coefficients, $\eta$ and $\zeta$, are the shear and bulk viscosity of the liquid, respectively. The viscosity coefficients have their origin in the approximate nature of the Navier-Stokes equations and in the particle-particle interaction [17]. The first equation in (1) is the continuity equation and states the mass conservation when sources or sinks are not present. The second equation in (1) is the force equation: the left hand side is the acceleration of a small volume of liquid subject to the internal forces (due to pressure, particle-particle interactions) and external forces $\left(F_{\text {ext }}=-\nabla V_{\text {ext }}\right)$. It is important to realize the approximate nature of these equations: in classical physics the very basic concept of particle density $n$ has a meaning only in a coarse-grained sense, i.e., with respect to volumes of the liquid small compared to the other relevant scales of the problem, but large to contain "enough" particles so that a continuum mechanics can be developed. In the opposite condition, one has to revert to the solution of the Newton equations of motion for each particle. Due to the continuous spatial nature of wave-functions, the above limitations do not pertain to Quantum Mechanics, for which a hydrodynamic description can be formulated exactly.

\section{HYDRODYNAMICAL FORMULATION OF QUANTUM MECHANICS}

Ever since the formulation of the Schrödinger equation of motion for complex wave-functions, there have been several attempts to formulate Quantum Mechanics in terms of classical quantities. The degree to which these attempts have been successful is still undecided, since the use of words like "particle", "trajectories", and "directions of propagation" is widespread in the modern scientific literature. One such attempt was made at the dawn of Quantum Mechanics, in 1926, by Madelung [17, 18] who showed that the Schrödinger equation for a single particle is exactly equivalent to a set of equations of motion for the particle density and "velocity". For this single-particle problem, the velocity is defined as the variation of the phase of the wave-function with position, and thus seems a mere mathematical tool [17]. An equivalent, but more transparent definition of this velocity field is

$$
v(r, t)=\frac{j(r, t)}{n(r, t)},
$$

where $j(r, t)$ is the current density. This definition is valid for the points $r$ for which $n(r, t) \neq 0$. It is remarkable that the equation of motion for this velocity is governed by the external forces, plus a "quantum mechanical" contribution, known as "Bohm stress tensor", that has not a classical counterpart [17]. Indeed, if we start from the Schrödinger equation for the wave-function, $\Psi$, of a particle in the presence of the external potential $V_{\text {ext }},(\hbar=e=1$ throughout this paper, where $e$ is the electron charge)

$$
i \partial_{t} \Psi(r, t)=-\frac{1}{2 m} \nabla^{2} \Psi(r, t)+V_{e x t}(r, t) \Psi(r, t)
$$

we can rewrite $\Psi(r, t)$ in terms of two real functions of time and position, $R$ and $S$, as

$$
\Psi(r, t)=R(r, t) e^{i S(r, t)}
$$

It is a simple exercise to show that, if one defines the density $n(r, t)$, and the velocity $v(r, t)$,

$$
\begin{aligned}
n(r, t)=R^{2}(r, t) & =|\Psi(r, t)|^{2}, \\
v(r, t) & =\frac{\nabla S(r, t)}{m},
\end{aligned}
$$

then the equations of motion

$$
\begin{array}{r}
\partial_{t} n(r, t)=-\nabla \cdot[n(r, t) v(r, t)], \\
m \partial_{t} v(r, t)=\frac{1}{2 m} \nabla\left(\frac{\nabla^{2} R(r, t)}{R(r, t)}\right)-m v(r, t) \cdot \nabla v(r, t)-\nabla V_{e x t}(r, t)
\end{array}
$$


hold. Eqs. (8) and (9) have a clear physical interpretation: The quantum mechanical system is equivalent to a fluid whose dynamics is governed by the Euler equation (9) subjected to the force exerted by the external potential [15], and an internal force whose origin is purely quantum mechanical. ${ }^{1}$ Moreover, the dynamics conserves the mass, i.e., the total probability, and then the continuity equation (8) holds [19]. The solution of the equations of motion (8) and (9) is equivalent to the solution of the Schrödinger equation. It is interesting to point out that the quantum mechanical force can be expressed in terms of the Bohm stress tensor,

$$
P_{i, j} \equiv-\frac{1}{4 m} n(r, t) \nabla_{i} \nabla_{j} \ln (n(r, t))
$$

and

$$
\frac{n(r, t)}{2 m} \nabla_{i}\left(\frac{\nabla^{2} R(r, t)}{R(r, t)}\right)=-\nabla_{j} P_{i, j}(r, t) .
$$

If one introduces the convective derivative the equations of motion (8) and (9) assume the well known form of the Navier-Stokes equations of motion

$$
\begin{array}{r}
D_{t} n(r, t)=-n(r, t) \nabla \cdot v(r, t), \\
m n(r, t) D_{t} v_{i}(r, t)=-\nabla_{j} P_{i, j}(r, t)-n(r, t) \nabla_{i} V_{e x t}(r, t) .
\end{array}
$$

Eqs. (12) and (13) are formally identical to the Navier-Stokes equations (1) for a classical fluid. However, unlike the Navier-Stokes equations which describe an approximate dynamics of the many-body classical fluid, Eqs. (12) and (13) are exactly equivalent to the Schrödinger equation: no approximation has been made in their derivation.

While this approach to Quantum Mechanics may appear as a simple attempt to recover a classical description of quantum phenomena, over the years it has proven to be a very useful tool to describe the dynamics of quantum systems in several contexts ranging from condensed matter physics to nuclear physics (see, e.g., [20], and references therein). More recently, we have shown that a hydrodynamic description of the electron flow in nanoscale systems leads to the prediction of novel phenomena, like the existence of a dynamical (viscous) resistance [21], turbulence [14, 22, 23, 24], and local electron heating and its effect on ionic heating [7, 9].

Here, we describe our hydrodynamical approach to transport in nanostructures. As a first step, we need to generalize the derivation of the equations of motion (12)-(13) to the case of a many-body interacting system. We follow closely the formalism presented in Refs. [14, 25]. (See also Ref. [26] for a general formulation of the dynamics of a manyparticle electron system.) We describe the dynamics of the system via a field creation (annihilation) operator $\psi^{\dagger}(r, t)$ $(\psi(r, t))$ which evolves in time following the Heisenberg equation of motion

$$
\begin{aligned}
i \partial_{t} \psi(r, t)= & -\frac{1}{2 m} \nabla^{2} \psi(r, t)+V_{e x t}(r, t) \psi(r, t) \\
& +\int d r^{\prime} \psi^{\dagger}\left(r^{\prime}, t\right) w\left(\left|r-r^{\prime}\right|\right) \psi\left(r^{\prime}, t\right) \psi(r, t),
\end{aligned}
$$

where the potential $w\left(\left|r-r^{\prime}\right|\right)$ describes the particle-particle interaction. We define the single-particle density operator via the usual definition, $\hat{n}(r, t)=\psi^{\dagger}(r, t) \psi(r, t)$ and the current density operator

$$
\hat{j}(r, t)=\frac{i}{2 m}\left[\left(\nabla \psi^{\dagger}(r, t)\right) \psi(r, t)-\psi^{\dagger}(r, t) \nabla \psi(r, t)\right] .
$$

It is lengthy but straightforward to show that these two operators follow the dynamics induced by the coupled equations of motion

$$
\begin{aligned}
\partial_{t} \hat{n}(r, t)= & -\nabla \cdot \hat{j}(r, t) \\
m \partial_{t} \hat{j}_{i}(r, t)= & -\hat{n}(r, t) \nabla_{i} V_{e x t}(r, t)-\nabla_{j} \hat{T}_{i, j}(r, t) \\
& -\psi^{\dagger}(r, t) \int d r^{\prime} \psi^{\dagger}\left(r^{\prime}, t\right) \nabla_{i} w\left(\left|r-r^{\prime}\right|\right) \psi\left(r^{\prime}, t\right) \psi(r, t)
\end{aligned}
$$

\footnotetext{
1 The first term on the right hand side of Eq. (9).
} 
where we have defined the kinetic stress tensor operator

$$
\begin{aligned}
\hat{T}_{i, j}(r, t)= & \frac{1}{2 m}\left[\nabla_{i} \psi^{\dagger}(r, t) \nabla_{j} \psi(r, t)+\nabla_{j} \psi^{\dagger}(r, t) \nabla_{i} \psi(r, t)\right. \\
& \left.-\frac{\delta_{i, j}}{2} \nabla^{2} \hat{n}(r, t)\right] .
\end{aligned}
$$

From the equations of motion for the operators, we get immediately the equations of motion for their expectation values

$$
\begin{aligned}
\partial_{t} n(r, t)= & -\nabla \cdot j(r, t) \\
m \partial_{t} j_{i}(r, t)= & -n(r, t) \nabla_{i} V_{e x t}(r, t)-\nabla_{j}\left\langle\hat{T}_{i, j}(r, t)\right\rangle \\
& -\int d r^{\prime} \rho_{2}\left(r, r^{\prime}, t\right) \nabla_{i} w\left(\left|r-r^{\prime}\right|\right)
\end{aligned}
$$

where $\rho_{2}\left(r, r^{\prime}, t\right)=\left\langle\psi^{\dagger}(r, t) \psi^{\dagger}\left(r^{\prime}, t\right) \psi\left(r^{\prime}, t\right) \psi(r, t)\right\rangle$. Another rather lengthy and involved calculation allows us to write the force density due to the particle-particle interaction as a second-rank tensor, provided the interactions are negligibly small at the boundary of the integration volume in equation (20). The result is [14, 25]

$$
\begin{aligned}
\nabla_{j} W_{i, j} & =-\frac{1}{2} \nabla_{j} \int d y \frac{y_{i} y_{j}}{|y|} \frac{d w(|y|)}{d|y|} \int_{0}^{1} d \lambda \rho_{2}(r+\lambda y, r-(1-\lambda) y, t) \\
& \equiv \int d r^{\prime} \rho_{2}\left(r, r^{\prime}, t\right) \nabla_{i} w\left(\left|r-r^{\prime}\right|\right)
\end{aligned}
$$

so that we arrive at the dynamical equation

$$
m \partial_{t} j(r, t)=-n(r, t) \nabla_{i} V_{e x t}(r, t)-\nabla_{j} P_{i, j}
$$

where we have defined

$$
P_{i, j}=W_{i, j}+\left\langle\hat{T}_{i, j}\right\rangle
$$

From here, by using the definition of convective derivative and re-scaling the particle momentum so that the stress tensor reads

$$
P_{i, j}=W_{i, j}+\left\langle\hat{T}_{i, j}\right\rangle-m n v_{i} v_{j}
$$

one obtains the equations of motion for the particle and current densities in a form identical to the single-particle equations of motion (12)-(13).

Like Eqs. (12) and (13), which, for a given initial condition, constitute a closed set, i.e., their solution is equivalent to the solution of the single-particle time-dependent Schrödinger equation, also their many-body counterpart, Eqs. (19) and (22) are equivalent to the solution of the many-body time-dependent Schrödinger equation. This equivalence is a direct consequence of the theorems of time-dependent density-functional theory [27, 28]. These theorems state that, given an initial condition, there exists a one-to-one correspondence between the time evolution of the particle density $n(r, t)$ and scalar potential $V_{\text {ext }}(r, t)$ applied to the quantum mechanical system. A similar correspondence holds between the current density $j(r, t)$ and an external vector potential $A(r, t)$ [27, 29, 30, 31], while the mapping does not generally exist between the current density and the external scalar potential [32]. The physical relevance of these theorems to our case is that the stress tensor $P_{i, j}(r, t)$ in (23) is a functional of either the density or the current density, i.e., $P_{i, j}(r, t)=P_{i, j}\left[n\left(r, t^{\prime}\right), t\right]$ or $P_{i, j}(r, t)=P_{i, j}\left[j\left(r, t^{\prime}\right), t\right]$ (with $\left.t^{\prime} \leq t\right)$. This implies that once the exact many-body stress tensor $P_{i, j}$ is known, one can, in principle, recover from the solution of Eqs. (19) and (22) full information on the many-body wave-function.

Needless to say, the exact stress tensor is unknown. However, starting from Eqs. (19) and (22) one can develop perturbation schemes to approximate the exact stress tensor [30, 33, 34], at least for the problem at hand, thus simplifying enormously the solution of the many-body problem. In the following, we will describe one of such approximation schemes for the present case of current flow in a nanojunction. We will derive an equation of motion for the stress tensor $P_{i, j}$ and show that it depends on the so-called three-particle stress tensor $P_{i, j, k}^{(3)}$, which in turn describes the way three particles interact. The derivation of an equation of motion for $P_{i, j, k}^{(3)}$ would bring us into the maze of a hierarchic set of equations for stress tensors that describe electron-electron interactions to all orders. We will show, however, that for the case at hand, we can truncate this hierarchy and obtain a closed equation for the stress tensor $P_{i, j}$. 


\section{VISCO-ELASTICITY OF THE ELECTRON LIQUID}

In parallel with the hydrodynamic description of Quantum Mechanics, a visco-elastic formulation of the dynamics of the electron liquid has been derived within linear-response theory. It has been realized that a certain class of low-energy, long-wavelength excitations of the electron liquid may be mapped into the dynamics of a visco-elastic medium [35]. The dynamics of this visco-elastic medium is described by an equation of motion for the current density given by (in linear response and $d$ dimensions, $d>1$ )

$$
\begin{aligned}
m n \partial_{t} v(r, t)= & {\left[\tilde{K}+\left(1-\frac{2}{d}\right) \tilde{\mu}\right] \nabla(\nabla \cdot v(r, t)) } \\
& +\tilde{\eta} \nabla^{2} v(r, t)-n(r, t) \nabla V_{e x t}(r, t)
\end{aligned}
$$

where $\tilde{K}$ and $\tilde{\mu}$ are two complex constants which depend on the electron density $n$. These complex constants are expressed in terms of the more familiar viscosities, $\zeta$ (bulk viscosity) and $\eta$ (shear viscosity) and elastic constants $K$ (bulk modulus) and $\mu$ (shear modulus) via the relations

$$
\begin{array}{r}
\tilde{K}(\omega)=K-i \omega \zeta, \\
\tilde{\mu}(\omega)=\mu-i \omega \eta,
\end{array}
$$

where $\omega$ is the frequency of the external perturbation used to excite the electron liquid.

The next step is then to express the visco-elastic coefficients of the liquid in terms of its microscopic properties, i.e., relate these quantities to the response functions. Here we only report the results that are relevant to the present work and refer the reader to Ref. [35] for an explicit derivation. We are only concerned with the DC (zero frequency) limit of the above quantities. By using an interpolation of the numerical results of mode-mode coupling theory [36] one finds the following density dependence of the zero-frequency shear viscosity (the bulk viscosity is identically zero in the same limit) [35]

$$
\frac{\eta}{n}=\frac{1}{60 r_{s}^{-3 / 2}+80 r_{s}^{-1}-40 r_{s}^{-2 / 3}+62 r_{s}^{-1 / 3}}
$$

in $3 \mathrm{D}$ and in $2 \mathrm{D}$ by

$$
\begin{aligned}
\left(\frac{\eta}{n}\right)^{-1}= & \left(\frac{r_{s}^{2}}{12 \pi} \ln \frac{2}{e r_{s}}+0.25 r_{s}^{2}\right)^{-1} \\
& +21 r_{s}^{-2}+23 r_{s}^{-1 / 2}+13
\end{aligned}
$$

where $r_{s}$ is the electron constant for the electron liquid with uniform density $n$ :

$$
r_{s} a_{B}=\left\{\begin{array}{cc}
(3 / 4 \pi n)^{1 / 3} & 3 \mathrm{D} \\
(1 / \pi n)^{1 / 2} & 2 \mathrm{D}
\end{array},\right.
$$

with $a_{B}$ the Bohr radius.

It is interesting to point out that specific confining potentials (e.g., an electron liquid in a quantum well) may make the approximations used to derive Eqs. (28) and (29) ill founded, leading to a peculiar behavior of the viscosity coefficients [37].

\section{HYDRODYNAMIC APPROACH TO TRANSPORT IN NANOSCALE SYSTEMS}

In this section we show that in the case of nanoscale systems the stress tensor can be approximated to a form similar to the classical Navier-Stokes one. This is due to the geometric constriction experienced by electrons flowing in the nanostructure which gives rise to a very short "collisional" time [38, 39]. The system we have in mind is some nanoscopic junction sandwiched between two mesoscopic or macroscopic leads (see Figure 11) and current is induced in the system by, e.g. polarizing the leads with a finite bias. In this regime, we show that one can truncate the infinite hierarchy of equations of motion for the electron stress tensor given in (23) to second order and thus derive quantum hydrodynamic equations. To realize how the simple presence of the junction has such a strong impact on the equation 


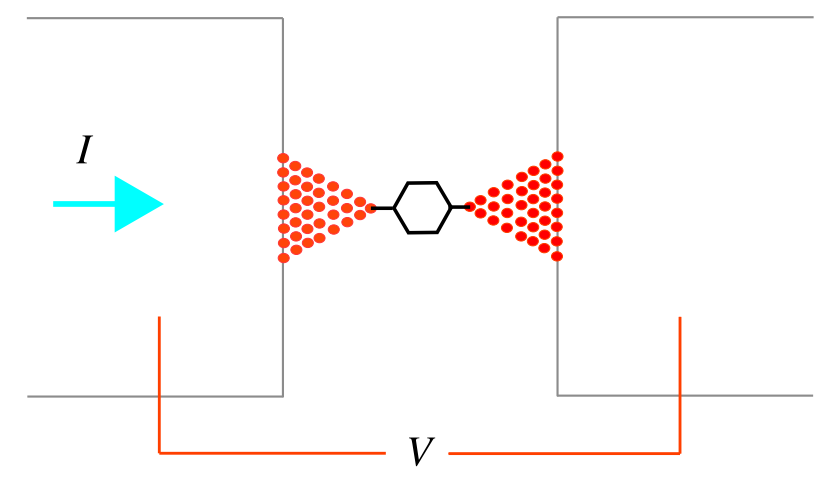

FIG. 1: Schematic of a nano-junction connected to two bulk electrodes. A steady current is flowing from one electrode to the other.

of motion of the current, one has to keep in mind that the former acts as a single impurity potential that cannot be avoided by the electron flow. This is different from the corresponding effect in bulk materials for which a certain density of impurities is necessary to have a finite resistance.

Let us then employ the quantum Boltzmann equation for the single-particle distribution function $f(r, p, t)$ (which can be derived from the time-dependent Schrödinger equation with standard techniques [40]) and show how the short collisional time induced by the nanostructure allows us to close the equations for the stress tensor. ${ }^{2}$ The quantum Boltzmann equation for the distribution function in a co-moving (Lagrangian) reference frame moving with velocity $v(r, t)$ is [14, 41]

$$
\begin{aligned}
I[f]= & D_{t} f(r, p, t)+\frac{p}{m} \nabla f(r, p, t)+e \nabla \varphi \frac{\partial f(r, p, t)}{\partial p}-p \cdot \nabla v \frac{\partial f(r, p, t)}{\partial p} \\
& -m D_{t} v \frac{\partial f(r, p, t)}{\partial p}
\end{aligned}
$$

where $I$ is the usual collision integral [40], $\varphi$ is the sum of the external potential and the Hartree part of the interaction potential. The collision integral contains two terms, one elastic and the other inelastic. In what follows, it is important to realize that both terms can drive the system toward a local equilibrium configuration.

From the quantum Boltzmann equation, we can derive the equation of motion for the moments of the distribution function. The general expression for the $m$ th moment is the $m$ th-rank tensor

$$
P_{i_{1}, \ldots, i_{m}}=\int d p p_{i_{1}} \ldots p_{i_{m}} f(p, r, t)
$$

The zeroth order is the single particle density, the first moment is the velocity field, and the second moment is the stress tensor we want to approximate. The equation of motion for the stress tensor contains a term proportional to the third moment $P^{(3)}$ :

$$
\begin{array}{r}
D_{t} P_{i, j}+P_{i, j} \nabla \cdot v+P_{i, k} \nabla_{k} v_{j}+P_{k, j} \nabla_{k} v_{i}+\nabla_{k} P_{i, j, k}^{(3)}= \\
\frac{1}{m} \int d p I[f] p_{i} p_{j} .
\end{array}
$$

We note that $P^{(3)}$ enters in (33) only through its spatial derivative. If the latter is small then the hierarchy can be truncated [14, 41]. From (33) we easily see that this derivative is small compared to the other terms whenever $\gamma=u /\left(L \max \left(\omega, \nu_{c}\right)\right) \ll 1$. Here $u$ is the average electron velocity, $L$ is the length of inhomogeneities of the liquid that give rise to scattering among three particles, $\omega$ the system proper frequency and $\nu_{c}$ the collision rate. The parameter $1 / L$ enters through the spatial derivative of $P^{(3)}, \omega$ from the frequency dependence of the interactions (in the DC limit of interest here $\omega \rightarrow 0), \nu_{c}$ through the collisional integral $I[f] \propto-\nu_{c}\left(f-f_{0}\right)$, where $f_{0}$ is the

\footnotetext{
${ }^{2}$ Clearly, for the definition of local equilibrium distribution to be valid any length scale entering the problem has to be larger than the system Fermi wavelength.
} 
equilibrium Fermi distribution. This derivative is indeed small for transport in nanostructures: When electrons move into a nano-junction they adapt to the given junction geometry at a fast rate, and approach to local equilibrium occurs at this fast rate even in the absence of electron interactions [38, 39]. This "relaxation" mechanism occurs roughly at a rate $\nu_{c}=(\Delta t)^{-1} \sim(\hbar / \Delta E)^{-1}$, where $\Delta E$ is the typical energy spacing of lateral modes in the junction. For a nano-junction of width $\ell$ we have $\Delta E \sim \pi^{2} \hbar^{2} / m \ell^{2}$ and $\Delta t \sim m \ell^{2} / \pi^{2} \hbar$. If $\ell=1 \mathrm{~nm}, \nu_{c}$ is of the order of $10^{15} \mathrm{~Hz}$, i.e., orders of magnitude faster than typical electron-electron or electron-phonon scattering rates. The condition $\gamma=u /\left(L \max \left(\omega, \nu_{c}\right)\right) \ll 1$ thus requires the length of inhomogeneities $L \gg 1 \mathrm{~nm}$, which is easily satisfied in nanostructures. Note instead that in mesoscopic structures this condition is not necessarily valid. In that case, the dominant relaxation rate $\nu_{c}$ is given by inelastic effects, i.e. it is of the order of $\mathrm{THz}$, so that for typical lengths of mesoscopic systems, $\gamma \approx 1$ in the DC limit. Nonetheless, the above condition could still be valid for high-frequency excitations, like plasmons, and/or very low densities, so that moments of the distribution of order higher than two are negligible.

By neglecting $\nabla_{k} P_{i, j, k}^{(3)}$ in (33) we can thus derive a form for $P_{i, j}$. Let us write quite generally the stress tensor $P_{i, j}$ as $P_{i, j}=\delta_{i, j} P-\pi_{i, j}$, where the diagonal part gives the pressure of the liquid, and $\pi_{i, j}$ is a traceless tensor that describes the shear effect on the liquid. From (33) we thus find that the tensor $\pi_{i, j}$ can be written as (in $d$ dimensions, $d>1)$

$$
\pi_{i, j}=\eta\left(\nabla_{i} v_{j}+\nabla_{j} v_{i}-\frac{2}{d} \delta_{i, j} \nabla_{k} v_{k}\right)
$$

where $\eta$ is a real coefficient (the viscosity) that is a functional of the density [41]. We point out that (34) is in fact a particular case of a general stress tensor with memory effects taken into account [30, 35, 42]. In our derivation this is the first non-trivial term of an expansion of the stress tensor in terms of the density and velocity field. Consequently the Navier-Stokes stress tensor in (34) can be seen as the first-order (non-trivial) contribution to the exact stress tensor of the electron liquid (see also [25, 30, 42]).

Using this stress tensor we finally get from (22) the generalized Navier-Stokes equations for the electron liquid in nanoscale systems

$$
\begin{aligned}
D_{t} n(r, t)= & -n(r, t) \nabla \cdot v(r, t), \\
m n(r, t) D_{t} v_{i}(r, t)= & -\nabla_{i} P(r, t)+\nabla_{j} \pi_{i, j}(r, t) \\
& -n(r, t) \nabla_{i} V_{e x t}(r, t) .
\end{aligned}
$$

Equations (36) are formally equivalent to their classical counterpart [15] [see Eq. (10] and thus describe also nonlinear solutions, i.e., the possibility to develop turbulence in the electron liquid in its normal state. In the following, we will consider only the case in which the liquid is in the laminar regime and incompressible so that the viscoelastic coefficients are spatially uniform. This latter approximation is practically satisfied in metallic quantum point contacts (QPCs) but needs to be relaxed in the case of QPCs with organic/metallic interfaces (see, e.g., 221]). In addition, for this case the Hartree potential is constant and its spatial derivative is thus zero. Therefore, (36) reduce to the Navier-Stokes equations for the density and velocity of a viscous but incompressible electron liquid

$$
\begin{aligned}
& D_{t} n(r, t)=0, \\
& \nabla \cdot v(r, t)=0, \\
& m n(r, t) D_{t} v_{i}(r, t)=-\nabla_{i} P(r, t)+\eta \nabla^{2} v_{i}(r, t)-n(r, t) \nabla_{i} V_{e x t}(r, t) .
\end{aligned}
$$

\section{HEAT EQUATIONS FROM HYDRODYNAMICS}

The above results allow us to treat heat generation and transport using a simplified hydrodynamic approach. In fact we know that the flow of a viscous fluid, as described by our formalism, generates internal friction and consequently an effective temperature distribution inside the system. Therefore, when a steady state has been reached, we can supplement the Navier-Stokes equations with an equation for the energy balance. In the process of heat production, we need to identify a heat source, a mechanism for the dissipation of this heat and, since the system is in a steady state, equate these two terms with the local entropy production. In a recent paper 7] we have developed this model obtaining the equation for the energy balance

$$
\pi_{i, j}(r) \partial_{j} v_{i}(r)+\nabla \cdot\left[k(r) \nabla T_{e}(r)\right]=c_{V}\left(T_{e}\right) v(r) \cdot \nabla T_{e}(r)
$$


where $T_{e}$ is the electronic temperature, $k(r)$ is the diffusion constant and $c_{V}$ is the specific heat at fixed volume of the electron gas. ${ }^{3}$ Eq. (37) can be either justified on physical grounds, or derived formally as high-order expansion of the many-particle stress tensor [25]. We also stress once more that in deriving this equation we have assumed that the flow of the electron liquid is laminar, i.e., we are far from the onset of a turbulent regime [14, 15]. Obviously, in writing Eq. (37) we have assumed that some thermodynamic quantities like temperature and entropy for an electron liquid flowing in a nanostructure can be defined. This is a much debated point, and obviously we do not have a general solution for it. However, here we argue that the electron temperature may be defined as the one ideally measured by a probe weakly coupled to the system and in local equilibrium with the latter [2]. While this operational definition may not be simple to realize in practice, we know from experiments that local heat generation due to current has a large effect on the stability of nanostructures [9].

From the form of Eq. (37) we can deduce a general relation between the applied bias and the electron temperature. To do this, we realize [7] that the electron fluid velocity, $v$, (which is generally smaller than the Fermi velocity [22]) responsible for the transport of current and heat is, in linear response, proportional to the bias $V$. 4 This simple proportionality, and the usual result that $k \propto c_{V}$, bring us to the general result

$$
T_{e}=\gamma_{e e} V
$$

where $\gamma_{e e}$ is a constant whose expression in terms of microscopic parameters of the electron liquid has been recently derived for a quasi-adiabatic connection between the leads and the nanojunction [7]

$$
\gamma_{e e}=1.16 \times\left(\frac{G}{n A_{c}}\right) \sqrt{\frac{d-1}{3 d} \frac{\eta}{\gamma}}
$$

where $G$ is the conductance of the system in the limit of zero bias, $A_{c}$ its cross section, $d$ is the dimensionality $(d>1)$. Moreover,

$$
\gamma=k_{F}^{2} k_{B}^{2} \lambda_{e} / 9
$$

in 3D, and $\gamma=\pi k_{F} k_{B}^{2} \lambda_{e} / 6$ in 2D [7], $k_{F}$ is the Fermi momentum, $k_{B}$ the Boltzmann constant, and $\lambda_{e}$ is the inelastic mean free path.

Interestingly, Eq. (38) can be obtained from general thermodynamic arguments, by comparing the energy dissipated in the transport process in the nanostructure (proportional to $V^{2}$ from Ohm's law), and the energy carried away by electrons (proportional to $T_{e}^{2}$ for small temperatures) [7].

\section{LOCAL ELECTRON HEATING}

In the case of a finite background temperature and in the absence of ionic heating, from our hydrodynamic theory, the local temperature of the electrons in the nanostructure is given by [7]

$$
T_{e}(V)=\sqrt{T_{0}^{2}+\gamma_{e e}^{2} V^{2}}
$$

where $V$ is the external bias, and $T_{0}$ is the electron temperature deep into the electrodes. If we now let the ions heat up, their effective local temperature is given by [7] (for values of the parameters such that the argument in the root is non-negative)

$$
T=\left(T_{0}^{4}+\gamma_{e p}^{4} V^{2}-\gamma_{e e}^{4} V^{4}\right)^{1 / 4}
$$

where $\gamma_{e p}$ can be expressed in terms of the physical parameters of the nanostructure [3, 43], and we have assumed that both the ions and the electrons are at the same background temperature $T_{0}$ deep into the electrodes. At zero background temperature and for negligible electron-electron interactions from the above equation we obtain the known result for the local ionic temperature [3, 7, 43]

$$
T \simeq \gamma_{e p} \sqrt{V}
$$

\footnotetext{
${ }^{3}$ For an electron gas at low temperature, $c_{V}=c_{P}$ since the correction is second order in temperature.

${ }^{4} \mathrm{~V}$ may be given by an external battery, or the potential due to a charge imbalance.
} 
Effect on conductance - We can now calculate the effect of local electron heating on the conductance of a nanostructure. We focus on the quasi-ballistic regime and we generalize Eq. (13) of Ref. 43] for the inelastic current in the presence of a finite electron temperature. ${ }^{5}$ We also consider one mode frequency $\omega$. We will generalize later to more modes. To take into account the effect of an effective local electron temperature on the inelastic current, one faces the calculation of terms with factors of the type $\int_{-\infty}^{\infty} d E f_{E}^{\alpha}\left(1-f_{E \pm \omega}^{\beta}\right)$, with $\alpha, \beta=\{R, L\}$ corresponding to electrons moving from either left or right, and $f_{E}^{\alpha}=\left(\exp \left(\left(E-\mu_{\alpha}\right) / k_{B} T\right)+1\right)^{-1}$ is the Fermi distribution with the difference between the electrochemical potentials equal to the bias, $\mu_{L}-\mu_{R}=V$. (Refer to [43] for additional details on the notation.)

We could provide a numerical calculation of the inelastic current. However, we are interested in an analytical expression and thus proceed as follows. We evaluate the above integrals in the Sommerfeld approximation and keep only the terms of zeroth order in the electron temperature (this is reasonable because the local electron temperature is generally a small quantity). This approximation brings us to the expression for the current flowing in the system

$$
I \simeq G_{e l} V-G_{e l} \frac{k_{B}}{\omega} \gamma_{I}\left(T_{0}^{4}+\gamma_{e p}^{4} V^{2}-\gamma_{e e}^{4} V^{4}\right)^{1 / 4} \frac{\log \left(e^{\beta(V-\omega)}+1\right)}{\beta},
$$

where $\gamma_{I}$ is the amplitude of the conductance drop at $V=V_{c} \equiv \omega$ for zero electron and phonon temperature, $G_{e l}$ is the elastic conductance at zero bias, and $\beta(V)=1 / k_{B} T_{e}(V)$ where $k_{B}$ is the Boltzmann constant. By differentiating Eq. (44) with respect to bias, and again keeping only the terms of zeroth order with respect the electron temperature, we arrive at

$$
\frac{G}{G_{e l}} \simeq 1-\frac{k_{B}}{\omega} \gamma_{I}\left(T_{0}^{4}+\gamma_{e p}^{4} V^{2}-\gamma_{e e}^{4} V^{4}\right)^{1 / 4}\left[\frac{1}{e^{\beta(V)(\omega-V)}+1}\right]
$$

To obtain this result, one also has to bear in mind that the approximations we make pertain to the energy region $V \simeq \omega$, thus $d(\beta(V)(V-\omega) / d V \simeq \beta(V)$. An expression for the conductance similar to Eq. (45) can be derived for the case of zero electron temperature [43], i.e., $\beta \rightarrow \infty$. Notice, however, that for consistency, one has to take this limit in the expression for the current (44) before taking the derivative with respect to the bias.

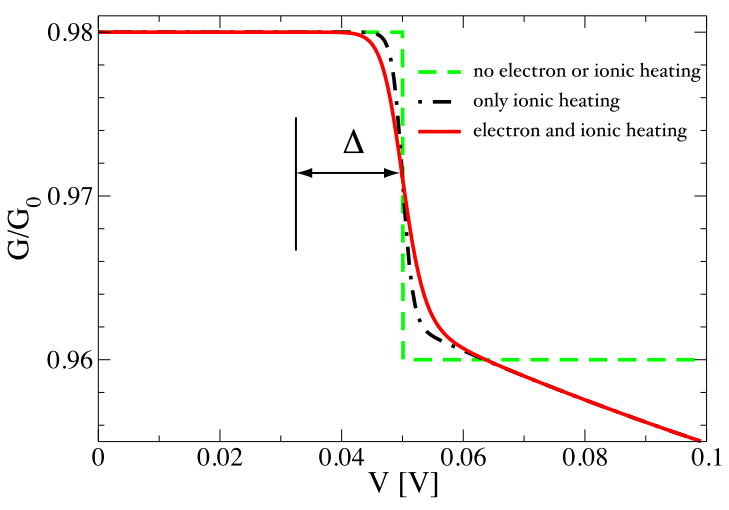

FIG. 2: Plot of $G / G_{0}$ as given by Eq. (45) where $G_{0}=2 e^{2} / h$. The solid (red) curve has been generated with the following parameters: $\gamma_{e e}=180 \mathrm{~K} / \mathrm{V}, \gamma_{e p}=600 \mathrm{~K} / \sqrt{\mathrm{V}}, G_{e l}=0.98 \times G_{0}, T_{0}=5 \mathrm{~K}, \gamma_{I}=0.02, \hbar \omega=0.05 \mathrm{eV}$. The dashed (green) line corresponds to the case of zero phonon and electron temperature $\left(\gamma_{e e}=\gamma_{e p}=0\right)$. The dashed-point (black) curve corresponds to the case when only the phonon heating is taken into account $\left(\gamma_{e e}=0\right)$. In the figure we also define the parameter $\Delta$ (see text).

An example of the effect of local ionic and electron heating on conductance is given in Fig. 2 (see also discussion below). In the absence of both effects (and at zero nominal background temperature) the conductance shows a simple step-like drop at the bias corresponding to the energy of the phonon mode. The ionic heating introduces a shoulder

\footnotetext{
${ }^{5}$ Note that a factor 2 is missing in Eqs. (7) and (8) of Ref. [43].
} 
at biases larger than the mode energy, while the electron heating broadens the conductance curve with an effective temperature larger than the nominal background temperature.

Comparison with experiments - To compare our results with available experimental data we consider a $\mathrm{D}_{2}$ molecule sandwiched between two Pt leads [13]. We focus on the predictions of our hydrodynamic theory on the local electron heating effect. Therefore, we do not attempt to do a full first-principles calculation of ionic heating, and take the relevant parameters from experiment. For the $\mathrm{D}_{2}$ molecule we consider a cross section of $\pi \times 1 \AA^{2}$, i.e., a circle with radius $\simeq 1 \AA$. The nominal electron temperature deep inside the electrodes is taken to be $T_{0}=5 \mathrm{~K}$. From the experimental results we have the frequency of the phonon mode $V_{c}=0.05 \mathrm{eV}$, the drop of the conductance

$$
\gamma_{I}=\left.\frac{\frac{d\left(I-I_{0}\right)}{d V}}{\frac{d I_{0}}{d V}}\right|_{V_{c}}=0.02,
$$

and the conductance at zero bias, $G_{e l}=0.984 G_{0}$. We use as fit parameters $\gamma_{e p}$ and evaluate $\gamma_{e e}$ from Eq. (39). In obtaining $\gamma_{e e}$ we have assumed an inelastic mean free path $\lambda_{e}$ of $1 \mu \mathrm{m}$, a value in line with the expectations for this system [4]. We have also assumed that the electron density that enters the local heating is the one of the chemical bonds between the $\mathrm{D}$ and $\mathrm{Pt}$ atoms. This density is estimated to be close to the Pt bulk density, $n=6.6 \times 10^{28} \mathrm{~m}^{-3}$ which gives the electron constant $r_{s} \simeq 3$. From these values, the electron viscosity $\eta$ and the constant $\gamma_{e e}$ are easily obtained from Eqs. (28) and (40), respectively: The electronic heating constant is predicted from Eq. (39) to be $\gamma_{e e}=180 \mathrm{~K} / \mathrm{V}$. This implies an effective electron temperature of about $10 \mathrm{~K}$ at the $\mathrm{D}_{2}$ junction at a bias of $50 \mathrm{mV}$. This temperature is higher that the nominal bulk temperature.

The ionic heating constant is found to be $\gamma_{e p}=405 \mathrm{~K} / \sqrt{V} .{ }^{6}$ This value can be compared with the corresponding $\gamma_{e p}$ for a $\mathrm{Au}$ point contact at small biases which is about $\gamma_{e p}^{A u}=170 \mathrm{~K} / \sqrt{V}[43]$. This means that the Pt- $\mathrm{D}_{2^{-}}$ Pt system heats up more than the Au QPC. For instance, at $0.1 \mathrm{~V}$ the ions of the $\mathrm{Pt}_{-} \mathrm{D}_{2}-\mathrm{Pt}$ junction have an average temperature of about $130 \mathrm{~K}$ while at the same bias the gold atoms heat up locally to about $54 \mathrm{~K}$. This larger temperature is reasonable since, while the conductance is similar for a $\mathrm{Au}$ point contact and $\mathrm{Pt}-\mathrm{D}_{2}-\mathrm{Pt}$, the $\mathrm{D}_{2}$ molecule is lighter than $\mathrm{Au}$ with a consequent increase of the electron-phonon coupling. In addition, the modes of the $\mathrm{D}_{2}$ molecule have lower probability to elastically scatter into the bulk modes of $\mathrm{Pt}$ - thus reducing lattice heat dissipation into the bulk electrodes - than the modes of a single Au atom into the bulk modes of Au. Both effects lead to a higher local ionic temperature. We thus expect the $\mathrm{Pt}-\mathrm{D}_{2}-\mathrm{Pt}$ junction to be more unstable under the same bias conditions than a Au point contact, i.e., we expect that the chain $\mathrm{Pt}_{-} \mathrm{D}_{2}-\mathrm{Pt}$ breaks, on average, at much smaller biases than Au point contacts due to heating effects.

The theoretical conductance containing both the local electron and ionic heating effects is reported in Fig. 3 , together with the experimental data. The qualitative agreement between theory and experiment is very good. It is interesting to note that the tail of the experimental data goes approximately as $V^{0.8}$, while the theory predicts $1-G / G_{0} \simeq V^{1 / 2}[3,5]$. It is important to realize, however, that at large biases, other effects such as current-induced forces and other structural instabilities may also contribute to the actual value of the conductance [45].

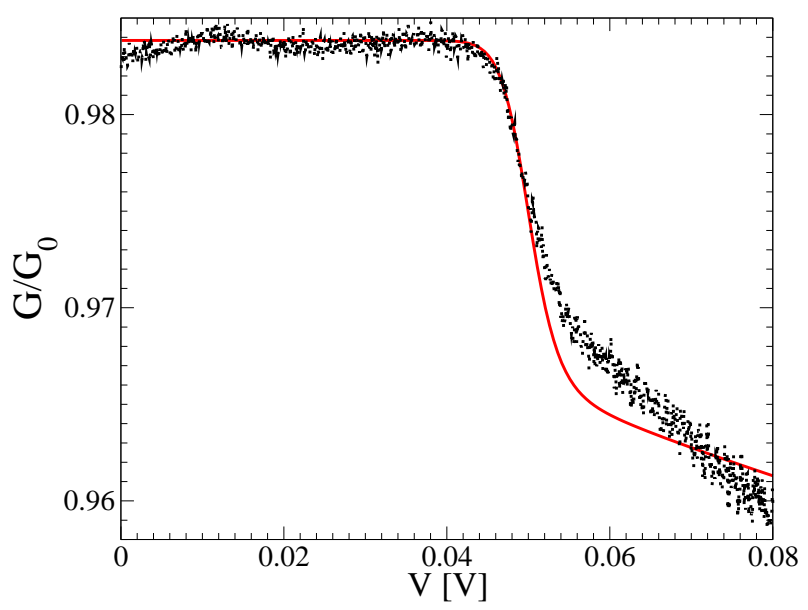

\footnotetext{
${ }^{6}$ This confirms that the ions heat more than the electrons at the same bias and our approximation that leads to Eq. (42) is justified.
} 


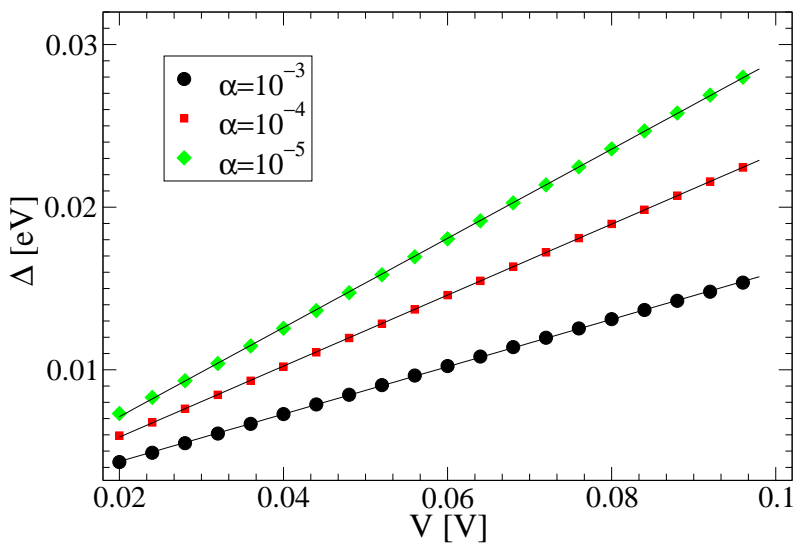

FIG. 4: $\Delta$ as a function of vibrational mode energy as defined in the text. We have tested the linear behavior for different values of the percentage $\alpha$ at which we calculate $\Delta$. The lines that connect the symbols are linear regressions.

FIG. 3: Comparison between the experimental data [13] and our theory [solid line, (red)] [14].

We have also performed a second fit, not shown here, using $\gamma_{e e}$ and $\gamma_{e p}$ as free parameters. The values for these parameters obtained from this second fit are close to those obtained from the theory and the one-parameter fit by less than $10 \%$ (we find the best fit for $\gamma_{e e}=200 \mathrm{~K} / \mathrm{V}$ ).

Inelastic conductance width - Let us now discuss how the width of the inelastic conductance around the vibrational mode increases with bias (see Fig. 22). This quantity can be directly measured and provides additional information on local electron heating. If the background temperature is zero, the local electron temperature increases linearly with bias as in Eq. (41). Let us define the quantity $\Delta$ as shown in Fig. 2. It is the energy distance between the middle drop of the conductance and the value at which the conductance assumes its purely elastic value within a ratio $\alpha=\left[G_{e l}-G\left(V_{c}\right)\right] / G_{e l}$ as indicated in Fig. 2, This quantity is plotted in Fig. 团 for different values of the vibrational mode energy and for a few values of $\alpha$, assuming that the vibrational energy is the only quantity allowed to vary. We conclude that the width $\Delta$ increases linearly with the vibrational energy to reflect the linear bias dependence of the local electronic temperature. A systematic experimental study of this quantity would thus provide more information on the electron heating phenomenon.

\section{DISCUSSION}

Our analysis in conjunction with the experimental data suggests that electrons heat up locally at the $\mathrm{Pt}-\mathrm{D}_{2}-\mathrm{Pt}$ junction. Our Eq. (41) also predicts that electrons cool down when lowering the bias. On the other hand, a constant electron temperature - above the background temperature - for all biases is difficult to understand on physical grounds, unless one assumes the existence of an external source of energy that keeps the electron hot even at zero bias.

Experimental data showing an electron temperature equal to the background temperature, i.e., negligible electron heating, may be consistent with the fact that the effective cross section "seen" by the electron liquid is the one of a Pt atom and not of a $\mathrm{D}_{2}$ molecule. ${ }^{7}$ If that were the case, the effective cross section would be 7 times larger than that of the $\mathrm{D}_{2}$ molecule, and since the electron temperature scales inversely proportional to the cross section (see Eq. (39) and Ref. [14]) the electron heating temperature would be lower than the background temperature. The conductance on the other hand is unlikely to be so sensitive to this cross section due to the extended nature of the Pt $d$-orbitals.

Further generalization of Eq. (45) to the case where many vibrational modes are present is possible. For example, it has been reported that a $\mathrm{H}_{2}$ molecule sandwiched between two $\mathrm{Pt}$ leads shows two fundamental vibrational frequencies at $48 \mathrm{meV}$ and $62 \mathrm{meV}$ [13]. If one assumes that scattering by these two modes is uncorrelated, a straightforward

\footnotetext{
7 This system can be thought of as a junction Pt-Pt-Pt with just one or few platinum atoms forming an effective QPC which is not significantly affected by the presence of the deuterium molecule.
} 

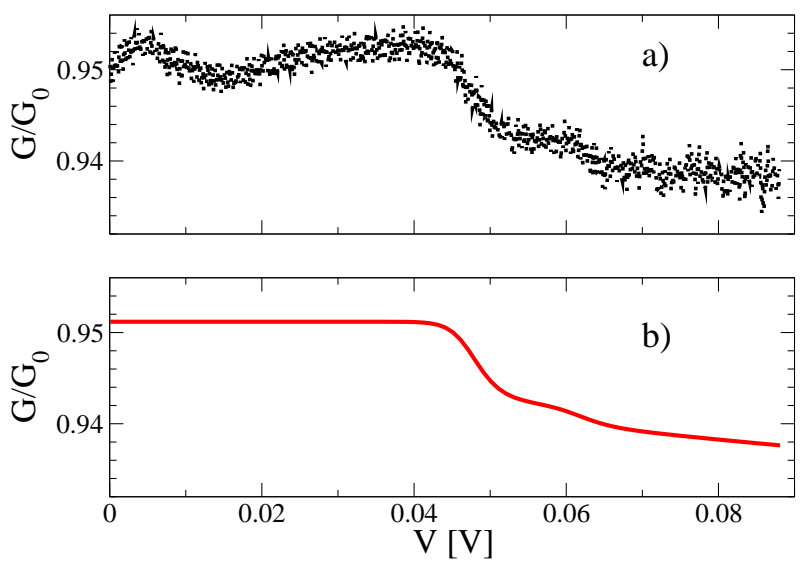

FIG. 5: a) Experimental results of the non-linear DC conductance of a $\mathrm{H}_{2}$ molecule between two electrodes. The two steps coming from the two phonon modes at 48 and $62 \mathrm{meV}$ are clearly visible, together with some unexpected structure at low bias. b) Plot of $G_{H_{2}}$ as a function of the external bias according to Eq. 47. In this plot, that is not a fit to the experimental data, we have used $\gamma_{I 1}=0.01, \gamma_{I 2}=0.002, \gamma_{e e}=180 \mathrm{~K} / \mathrm{V}, \gamma_{e p, 1}=\gamma_{e p, 2}=400 \mathrm{~K} / \sqrt{\mathrm{V}}$. The other parameters are the same as in Fig. 3.

generalization of Eq. (45) leads to

$$
\begin{aligned}
\frac{G_{H_{2}}}{G_{e l}} \simeq & 1-\frac{k_{B}}{\omega_{1}} \gamma_{I 1}\left(T_{0}^{4}+\gamma_{e p, 1}^{4} V^{2}-\gamma_{e e}^{4} V^{4}\right)^{1 / 4}\left[\frac{1}{e^{\beta(V)\left(\omega_{1}-V\right)}+1}\right] \\
& -\frac{k_{B}}{\omega_{2}} \gamma_{I 2}\left(T_{0}^{4}+\gamma_{e p, 2}^{4} V^{2}-\gamma_{e e}^{4} V^{4}\right)^{1 / 4}\left[\frac{1}{e^{\beta(V)\left(\omega_{2}-V\right)}+1}\right],
\end{aligned}
$$

where $\omega_{1}$ and $\omega_{2}$ are the two vibrational frequencies and we have taken into account the possibility that the two coupling constants $\gamma_{e p, 1}$ and $\gamma_{e p, 2}$, and the two amplitudes of the conductance drops $\gamma_{I 1}$ and $\gamma_{I 2}$ be different. A plot of $G_{H_{2}}$ is reported in Fig. 5 as a function of the external bias along with the experimental data. Since the cross section for $\mathrm{D}_{2}$ and $\mathrm{H}_{2}$ is essentially the same, and the electron heating does not depend on the mass of the ions, our estimate of $\gamma_{e e}$ holds for $\mathrm{H}_{2}$ as well. Our results are again in qualitative agreement with the available experimental data [13], although our theory might be not sufficient to quantitatively describe all the experimental findings. Indeed, our fit in this case has failed in producing any sensible result for the constants $\gamma_{e e}, \gamma_{e p, 1}$ and $\gamma_{e p, 2}$ : the large fluctuations of the experimental data, especially in the region of small bias and close to the phonon modes energies do not allow for a systematic fit of the data with the theory. Finally, it is interesting to note that a value of $\gamma_{e p}$ similar to the one we have obtained for the $\mathrm{D}_{2}$ molecule gives a reasonably good agreement between theory and experiment also for the $\mathrm{H}_{2}$ molecule. This seems to suggest that the longitudinal modes of the bonds between the $\mathrm{H}$ and $\mathrm{Pt}$ atoms are mainly responsible for the local ionic heating of the $\mathrm{Pt}-\mathrm{H}_{2}-\mathrm{Pt}$ junction, and similarly the longitudinal modes of the bonds between the $\mathrm{D}$ and $\mathrm{Pt}$ for the $\mathrm{Pt}-\mathrm{D}_{2}-\mathrm{Pt}$ junction. We expect that such modes are slightly affected by the change of mass of the smaller atom in the bond. Clearly, more theoretical and experimental work in this direction is necessary.

\section{CONCLUSIONS}

We have discussed a novel hydrodynamic approach to transport that allows the description of charge and heat flow in terms of the single-particle density and velocity field of the electron liquid 14]. The theory allows us to make predictions about the electron flow past a nanostructure and its dependence on the external bias (or the current). One such prediction is the heating of electrons locally at the nano-junction 7]. Here we have considered the measurable consequences of this effect on the inelastic conductance which shows a broadening at the inelastic step larger than the one expected from the background nominal temperature. We have compared our theory with available experimental results [13] and found a reasonable quantitative agreement for the case of a $\mathrm{D}_{2}$ molecule between two Pt leads. For the case of a $\mathrm{H}_{2}$ molecule between the same leads our theory is only in qualitative agreement with the experimental findings. We also predict that the width of the inelastic conductance step should increase linearly with bias, a fact that can be tested experimentally. 


\section{Acknowledgments}

We acknowledge financial support from the Department of Energy grant DE-FG02-05ER46204. We thank the authors of Ref. [13] for the use of their data and useful discussions.

\section{References}

[1] Di Ventra M, Evoy S and Heflin J R (eds) 2004 Introduction to Nanoscale Science and Technology (Springer)

[2] Di Ventra M 2008 Electrical transport in nanoscale systems (Cambridge University Press, in press)

[3] Todorov T 1998 Phil. Mag. B 77965

[4] Segal D and Nitzan A 2002 J. Chem. Phys. 1173915

[5] Chen Y C, Zwolak M and Di Ventra M 2003 Nano Lett. 31961

[6] Chen Y C, Zwolak M and Di Ventra M 2005 Nano Lett. 5621

[7] D'Agosta R, Sai N and Di Ventra M 2006 Nano Lett. 62935

[8] Huang Z, Xu B Q, Chen Y C, Di Ventra M and Tao N J 2006 Nano Lett. 61240

[9] Huang Z, Chen F, D'Agosta R, Bennett P A, Di Ventra M and Tao N J 2007 Nature Nanotech. 2698

[10] Pecchia A, Romano G and Di Carlo A 2007 Phys. Rev. B 75035401

[11] Tsutsui M, Kurokawa S and Sakai A 2007 Applied Physics Letters 90133121

[12] Tsutsui M, Kurokawa S and Sakai A 2006 Nanotechnology 175334

[13] Djukic D, Thygesen K S, Untiedt C, Smit R H M, Jacobsen K W and van Ruitenbeek J M 2005 Phys. Rev. B (R) 71 161402

[14] D'Agosta R and Di Ventra M 2006 J. Phys.: Cond. Matt. 1811059

[15] Landau L D and Lifshitz E M 1987 Fluid mechanics (Course of theoretical physics vol 6) (Pergamon Press)

[16] Goldstein S 1965 Modern Developments in Fluid Dynamics vol I (New York: Dover Publications)

[17] Ghosh S K and Deb B M 1982 Phys. Rep. 921

[18] Madelung E 1926 Z. Physik 40322

[19] Sakurai J J 1994 Modern Quantum Mechanics 2nd ed (Addison Wesley)

[20] Kan K K and Griffin J J 1977 Phys. Rev. C 151126

[21] Sai N, Zwolak M, Vignale G and Di Ventra M 2005 Phys. Rev. Lett. 94186810

[22] Sai N, Bushong N, Hatcher R and Di Ventra M 2007 Phys. Rev. B 75115410

[23] Bushong N, Gamble J and Di Ventra M 2007 Nano Lett. 71789

[24] Bushong N, Pershin Y V and Di Ventra M 2007 Phys. Rev. Lett. 99226802

[25] Tokatly I V 2005 Phys. Rev. B 71165104

[26] Martin P C and Schwinger J 1959 Phys. Rev. 1151342

[27] Marques M A L, Ullrich C A, Nogueira F, Rubio A, Burke K and Gross E K U (eds) 2006 Time-Dependent Density Functional Theory (Lecture Notes in Physics vol 706/2006) (Springer Berlin / Heidelberg)

[28] Runge E and Gross E K U 1984 Phys. Rev. Lett. 52997

[29] Ghosh S K and Dhara A K 1988 Phys. Rev. A 381149

[30] Vignale G and Kohn W 1996 Electronic density functional Theory: recent progress and new directions ed Dobson J F, Vignale G and Das M P (N.Y.: Plenum) p 199

[31] Di Ventra M and D'Agosta R 2007 Phys. Rev. Lett. 98226403

[32] D'Agosta R and Vignale G 2005 Phys. Rev. B 71245103

[33] Vignale G and Kohn W 1996 Phys. Rev. Lett. 772037

[34] Vignale G, Ullrich C A and Conti S 1997 Phys. Rev. Lett. 794878

[35] Conti S and Vignale G 1999 Phys. Rev. B 607966

[36] Nifosi R, Conti S and Tosi M 1998 Phys. Rev. B 5812758

[37] D'Agosta R, Di Ventra M and Vignale G 2007 Phys. Rev. B 76035320

[38] Di Ventra M and Todorov T 2004 J. Phys. Cond. Matt. 168025

[39] Bushong N, Sai N and Di Ventra M 2005 Nano Lett. 52569

[40] Kadanoff L P and Baym G 1962 Quantum Statistical Mechanics (N.Y.: Benjamin)

[41] Tokatly I V and Pankratov O 1999 Phys. Rev. B 6015550

[42] Tokatly I V 2005 Phys. Rev. B 71165105

[43] Chen Y C and Di Ventra M 2005 Phys. Rev. Lett. 95166802

[44] Pothier H, Guéron S, O B N, Esteve D and Devoret M H 1997 Phys. Rev. Lett. 793490

[45] Yang Z, Chshiev M, Zwolak M, Chen Y C and Di Ventra M 2005 Phys. Rev. B 71041402 